\title{
The New Model of Financial Market Regulation: How to Limit the Liquidity Crisis
}

\author{
Daniele Manini, Paola Pisano, Marco Pironti, and Marco Gribaudo
}

\begin{abstract}
The current financial crisis has shown the fragility of the regulation authorities especially in the managing of both Large complex financial institutions and toxic financial assets. This paper aims to propose a new form of financial market regulation based on the visibility of the risk rate of financial products to limit the liquidity problematic in presence of discontinues events. A qualitative result obtained by Mean Field Analysis methodology is illustrated to show the applicability of our proposal.
\end{abstract}

Index Terms-Toxic asset, liquidity crisis, visibility, main field analysis.

\section{INTRODUCTION}

The current financial crisis has shown the fragility of the regulation authorities or rather of the three levels of European committee (Cebs, Cesr and Ceiops). The regulation problematic were found especially in the managing of both Large complex financial institutions (Lcfi) and toxic financial assets.

The explanations of these problematic are to find in the lack of information about the actors and products that interact in the market. A research done by the central banks [9] before the financial 2007 crisis showed that the Lcfi problems are not the big dimension (in term of geographical extension or the asset) but the complexity (or rather the difficult to know the effective risk by supervisor), the interconnection (or rather the big probability to generate a ripple effect) and exhibition to common factors (that amplify the reaction creating a endogenous risk) [8].

While the actors regulation has been widely discussed (e.g. see [7]), the asset regulation has been neglected due to the complex classification of the financial products. This complexity depends on the different risk types lying inside the asset: the legal risk linked to the legal characteristics of products; the volatility risk linked to the product performances and the liquidity risk linked to the likelihood to sell the products.

It would be useful to create an authority organization that will declare the risk rate of the products in order to both test products functionality before their distribution, and to provide a kind of product licence, exactly like happens in the pharmaceutical industry. Moreover the authority will follow the products in all market life declaring their possible state switch in toxic asset caused for example by

Manuscript received July 20, 2012; revised August 30, 2012.

D. Manini is with the Computer Science Department of University of Torino, Torino, ITALY (e-mail: manini@di.unito.it).

P. Pisano and M. Pironti are with the E-Business Lab of University of Torino, Torino, ITALY.

M. Gribaudo is with the Polytechnic of Milano, Milano, ITALY. the actors who acquire them or by the quantity gained.

Getting inspiration from these scheme, the authors present a new regulation framework focused on both the financial assets and on their visibility for restraining the financial liquidity crisis. The framework is based on the idea that if the risk inside the asset is visible then it is possible to boundary the impact of the toxic products instead of limit the liquidity throughout the financial market. The study of the asset regulation is done developing a model through the Mean Field Analysis approach [5]. This approach permits to intuitively describe a systems characterized by a large number of interacting objects, such as financial products. Thanks to its multilevel structure it allows to easily create different scenario in which analyze the model. After an overview on the financial assets (Section II), the paper will describe the modelling approach (Sections III and IV). A qualitative result (Section V) shows that in visibility condition the financial liquidity crisis is narrow only on toxic product and not on the entire financial system.

\section{THE OVERVIEW ON THE FinANCIAL ASSET}

Normally the financial product suffers from different risk types: the legal risk, volatility risk and the liquidity risk. A common financial product can become a toxic product under a set of conditions depending on the market situation (for example in crisis period) or on the actor that buys it or on the quantity bought. A toxic asset is any asset that has a current market value significantly below its book value. Further, the market for a toxic asset is very illiquid. It is virtually impossible to sell a toxic asset at a reasonable price, if at all. Toxic asset was a term frequently used during the banking crisis of 2007-2009 [1]-[4].

In this case, the term toxic asset referred to collateralized debt obligations, credit default swaps, or mortgage-backed securities.

A mortgage-backed security became a toxic asset as a result of the following process. First, with the sharp decrease in house prices underlying the mortgage-backed security, its value dropped dramatically. Second, the holder of this mortgage-backed security couldn't find a buyer. Indeed, unless house prices went back to inflated high prices underlying most of those mortgage-backed securities, the purchaser of this toxic asset was guaranteed to lose money. Sharp decrease in value and illiquid trading markets are two key features of a toxic asset.

When a financial product becomes a toxic asset lead a set of problems:

- according to market observers, these toxic assets could not be easily valued and were therefore illiquid; 
- banks with many such assets could not sell them easily to raise funds necessary for lending;

- the authorities like the Treasury and the Fed have to respond with a combination of policy actions including asset purchases, loan guarantees, and equity injections.

The presence of an authority able to follow the financial products from the creation can limit the liquidity problem.

The product - with the detail of its risks (especially liquidity one) - will be tagged by the authority during its life on the market in order to keep updated its risk status.

This type of control will permit to circumscribe the crisis identifying the toxic product and consequently not extending the liquidity problematic to all the financial market.

The following section shows the Mean Field Analysis formalism and the Section IV illustrates the process used to model the proposed asset regulation through this methodology.

\section{MEAN FIELD ANALYSIS APPROACH}

The Mean Field Model is a representation that describes the behavior of a system as a collection of a large number of interacting objects.

\section{A. Formal Specification}

Objects are divided into classes: all the objects belonging to a given class have exactly the same behavior characterized by exactly the same parameters. If two objects perform the same actions at different rates, they must belong to different classes. Objects might be influenced by the distribution of the other objects in the system. Each object is modeled by a Continuous Time Markov Chain (CTMC), whose transition rates may depend on the state of the whole system. A CTCM is a mathematical description of a simple Stochastic process, characterized by a state, whose dynamic behavior depends only on its current state. In order to ease the description of complex systems, classes are further grouped into meta-classes. All the classes that derive from the same meta-class are characterized by the same structure, but different rates. The number of objects in every class changes dynamically: new objects might be formed at a given rate (expressed as quantity of new objects created per unit of time), and each object has an exponentially distributed maximum lifetime. More formally, we call the Mean Field Model M, a tuple:

$$
\mathrm{M}=(\mathrm{MC}, \mathrm{OC})
$$

where $\mathrm{MC}=\left\{\mathrm{mc}^{(1)}, \ldots, \mathrm{mc}^{(\mathrm{k})}\right\}$ is a set of $\mathrm{k}$ meta-classes and $O C=\left\{\mathrm{OC}^{[1]}, \ldots, \mathrm{oc}^{[\mathrm{m}]}\right\} \quad$ is a set of $\mathrm{m}$ object classes. Each meta-class $\mathrm{mc}^{(\mathrm{i})} \mathrm{mc}$ is in turn defined by a tuple:

$$
m c^{(i)}=\left(c^{(i)}, n^{(i)}, L^{(i)}, \Lambda^{(i)}, C^{(i)}, b^{(i)}, D^{(i)}\right)
$$

where $\mathrm{c}^{(\mathrm{i})}$ is a label corresponding to the name of the metaclass, $n^{(i)}$ is the number of states of the CTMC, $L^{(i)}=\left\{l^{(i)}\right\}$ is a set of labels (the names of the states) and $\Lambda^{(\mathrm{i})}=$ $\left\{\lambda_{1}^{(\mathrm{i})}, \ldots, \lambda_{\mathrm{p}_{\mathrm{i}}}^{(\mathrm{k})}\right\}$ is a set of formal parameters. $\mathrm{C}^{(\mathrm{i})}=\left|\mathrm{c}_{\mathrm{ul}}^{(\mathrm{i})}\right|$ is the $n(i) \times n(i)$ infinitesimal generator of the CTMC where $c_{u l}^{(i)}$ is the transition rate from state $u$ to state $1 . b^{(i)}=\left|b_{l}^{(i)}\right|$ is the size $n(i)$ birth vector: its element $b_{l}^{(i)}$ represents the rate at which new objects are created in state $1 . \mathrm{D}^{(\mathrm{i})}=$ $\operatorname{diag}\left(\mathrm{d}_{1 \mathrm{l}}^{(\mathrm{i})}\right)$ is a $\mathrm{n}(\mathrm{i}) \times \mathrm{n}(\mathrm{i})$ diagonal matrix, such that $1 /$ $\mathrm{d}_{\mathrm{ll}}^{(\mathrm{i})}$ represents the mean exponential lifetime of an object in state 1 . The entries of $\mathrm{C}^{(\mathrm{i})}, \mathrm{b}^{(\mathrm{i})}$ and $\mathrm{D}^{(\mathrm{i})}$ may depend on the actual values assigned to the parameters $\Lambda$. An object class ${ }_{\text {oc }}{ }^{[j]}$ is also a tuple:

$$
\mathrm{oc}^{(\mathrm{j})}=\left(\mathrm{o}^{(\mathrm{j})}, \mathrm{c}^{(\mathrm{j})}, \Gamma^{(\mathrm{j})}, \mathrm{N}^{(\mathrm{j})}, \pi_{0}^{(\mathrm{j})}\right)
$$

where $o^{(j)}$ is a label representing the name of the class; $c^{(j)}$ is name of the meta-class from which the class derives; $\Gamma^{(\mathrm{j})}=$ $\left\{\gamma_{1}^{[j]}, \ldots, \gamma_{p_{i}}^{[j]}\right\}$ is the set of actual parameters assigned to each of the formal parameters of the meta-class defined by $\Lambda^{(\mathrm{i})} ; \mathrm{N}^{[\mathrm{j}]}$ is the initial number of objects; $\pi_{0}^{[\mathrm{j}]}$ is a probability vector of size $n^{[j]}$ that defines the initial state probability for the objects belonging to this class. We define $\mathrm{n}^{\mathrm{j}]}$ as the number of states of class $\mathrm{j}$ inherited from its metaclass, that is $n^{[j]}=n^{\text {(meta -class of } j)}$. Note that we use round brackets in superscripts for elements corresponding to metaclasses and square brackets to denote elements belonging to classes. The value of each actual parameters can depend on the distribution of the number of objects among the states of all the classes that compose the model.

\section{B. Solution}

While the state space growths exponentially in conventional compositional approaches, our mean field based methodology provides approximations of the system that scales linearly with respect to the number of objects. Thanks to the previous assumptions, the solution of the model can be approximated using the Mean-Field analysis technique [6], following the results proposed in [5].

In particular the counts of the number of objects in each state are approximated by continuous variables, that are expressed by means of a set of ordinary differential equations. The solution of such equations, which is obtained using a suitable numerical algorithm, describes the evolution of the model. Initially, object classes are instantiated: matrix $\mathrm{C}^{[\mathrm{j}]}(\cdot)$, vector $\mathrm{b}^{[\mathrm{jj}]}(\cdot)$ and matrix $\mathrm{D}^{[\mathrm{j}]}(\cdot)$ are computed for each $\mathrm{oc}^{[\mathrm{j}]}$ by inserting the actual parameters $\Gamma^{[\mathrm{j}]}$ in the definitions of $\mathrm{C}^{(\mathrm{i})}, \mathrm{b}^{(\mathrm{i})}$ and $\mathrm{D}^{(\mathrm{i})}$. We call $\mathrm{N}^{[\mathrm{jj}]}(\mathrm{t})=\left|\mathrm{N}^{[\mathrm{j}]}{ }_{1}(\mathrm{t})\right|$ a vector of size $\mathrm{n}^{[\mathrm{j}]}$, whose element $\mathrm{N}^{[\mathrm{j}]}{ }_{1}(\mathrm{t})$ represents the number of objects of class $\mathrm{j}$ in state 1 at time $t$. Formal parameters can depend on the number of objects in each state, and thus we have $\mathrm{C}^{[\mathrm{j}]}\left(\mathrm{N}^{[1]}(\mathrm{t}), \ldots, \mathrm{N}^{[\mathrm{m}]}(\mathrm{t})\right), \mathrm{b}^{[\mathrm{j}]}\left(\mathrm{N}^{[1]}(\mathrm{t}), \ldots, \mathrm{N}^{[\mathrm{m}]}(\mathrm{t})\right), \mathrm{D}^{[\mathrm{j}]}\left(\mathrm{N}^{[1]}(\mathrm{t})\right.$, $\left.\ldots, N^{[m]}(t)\right)$. The evolution of the system can then be studied solving for $\mathrm{j}=1$..m:

$$
\frac{d N(t)}{d t}^{[j]}=N^{[j]}(t)\left[C^{[j]}(t)-D^{[j]}(t)\right]+b^{[j]}(t)
$$

with $\mathrm{N}^{[\mathrm{j}]}(0)=\mathrm{N}^{[\mathrm{jj}]} \pi_{0}{ }^{[\mathrm{j}]}$. The derivation of Eq. (4) can be summarized as follows. To simplify the presentation we drop the [j] superscript and the state dependencies $(\cdot)$. The number of objects of class $j$ in state 1 at time $t+\Delta t$ can be approximated by:

$$
N_{l}(t+\Delta t) \approx
$$

$N_{l}+\sum_{u \neq l} N_{u}(t)_{c_{u l}} \Delta t-N_{l} \sum_{u \neq l} c_{l u}(t) \Delta t-N_{l}(t) d_{u} \Delta t+$ $b_{l} \Delta t$ 
The second and third terms on the r.h.s. of Eq. (5) represent objects entering and leaving state 1 , while the last two terms consider the death and the birth of objects. By applying the definition $c_{l l}=\sum_{u \neq l} c_{l u}$, rearranging the terms, and dividing by $\Delta \mathrm{t}$ we obtain:

$$
\frac{N_{l}(t+\Delta t)}{\Delta t} \approx \sum_{u} N_{u}(t)_{c_{u l}}-N_{l}(t) d_{u}+b_{l}
$$

Eq. (4) can be obtained by letting $\Delta t \rightarrow 0$, and using vector notation.

TABLE I: THE MODEL ClASSIFICATION.

\begin{tabular}{|l|l|}
\hline CLASS & META-CLASS \\
\hline Crisis Level & Financial Scenario \\
\hline Visibility & Regulation \\
\hline Product A & Product \\
\hline Product B & Product \\
\hline Product C & Product \\
\hline
\end{tabular}

\section{THE MODEL}

In this section we first describe the financial market framework we considered, then we provide the process adopted to develop the corresponding mean-field analysis based model.

\section{A. Description}

The model has been creating considering the sequent milestones:

1) the product. We define three products: $\mathrm{A}$ is a product that under established conditions can become toxic; $B$ and $C$ are products with different behaviour.

2) the actors. The products can be exchanged between two entities: from the institutions, that have an intermediary role, to the investors.

3) the authority. We suppose to have a new entity that monitors the risk level of financial products.

In the model are considered three different financial scenarios: level 1 defines a normal economic/financial situation without any crisis; level 2 accounts for an incoming crisis; level 3 represents a stronger crisis than the one in level 2. The products are sold with different rates according to the financial scenario, i.e. higher the crisis level and lower the exchange rate. Under these assumptions there is a liquidity crisis that invests all the financial market and consequently all the assets.

By introducing the supervision of an authority that monitors the risk of the products, the effects on the market are different. Nevertheless the crisis reduces the capacity of investment for all the products, the regulator activity performed by the authority allows to limit the exchange rate of the asset became toxic. Otherwise the no toxic assets are not affected by the liquidity crisis, and they are still exchanged even with a lower rate.

The mean field methodology used for outline this framework regulation can be summarized in three steps. First, we identify the different types of entities that compose the system and we abstract their behaviour into metaclasses. Second, we define the Markov chains and the formal parameters of the meta-classes. Finally, we define a class for each type of entity. Each class is derived from a meta-class by assigning appropriate rates to the formal parameters

The solution computed by the Mean Field Analysis Model provides the time evolution of the number of objects for each class. The goal of this work is to observe the number of asset units exchanged in different scenarios and in presence/absence of the regulation policy, hence the attention is focused on the number of objects representing the products purchased by investors.

\section{B. First-Step: Classes and Meta-Classes Identification}

Since the classes represent the actors of our model, we first identify the entities that characterize this phenomenon, and we look for similarities to abstract their behaviour and to define an appropriate number of meta-classes. We decide to define our model through five actors grouped in three meta-classes (see table 1).

The metaclass Financial Scenario has just one class that is Level used to define the crisis status of the market. We decide to consider three different crisis levels:

- Level 1: defines a normal financial situation;

- Level 2: defines a low level of financial crisis;

- Level 3: defines a high level of financial crisis.

The metaclass Regulation has one class that is Visibility used to denote the presence/absence of the visibility of the toxic assets.

The metaclass Product has three classes that are Product $A$, Product $B$ and Product $C$ used to define different products with respect to their risk degree. Product $A$ is characterized by a high degree of risk whereas Product $B$ and Product $C$ have lower degree. Each of these classes class is characterized by a high number of objects that represent the asset units inside the market.

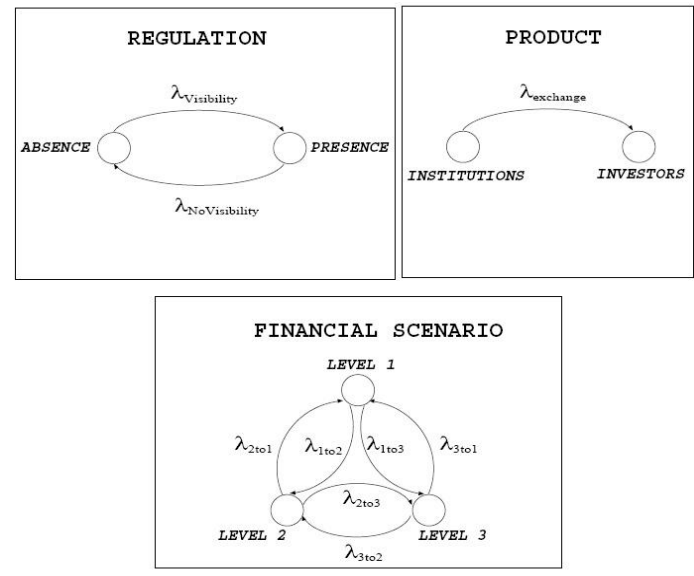

Fig. 1. The markov chains of regulation, product and financial scenario meta-classes.

\section{Second-Step: Meta-Class Specification}

Now we define the Markov chains (depicted in Fig. 1) corresponding to the meta-classes identified before.

Financial Scenario can be in three states each one representing the three different market status described before. The transition among the states depends on the rates $\lambda$ set in an arbitrary way. Note that each state can be reached by the others, for instance the transition from level 2 to level 3 happens with rate $\lambda_{2 \text { to } 3}$.

The Visibility can be in two states, Presence or Absence, that describe the regulation policy performed by the market authority. The transitions among the two states depend on 
the rates $\lambda$ set in an arbitrary way.

The Product has two states, Institutions and Investors, that describe whether the products are in the institution or in the investor portfolio. The exchange rate from institutions to investors is defined by $\lambda_{\text {exchange }}$.

All the meta-classes presented above can be formally expressed using the tuple reported in (2).

\section{Third-Step: Classes and Parameters speCification}

The crucial phase of this work is the computation of the rates that determine the relations and the interactions among all class objects of the model. The formal rates (depicted in Fig. 1) must be instantiated for each class. As mentioned above the parameters of classes derived from meta-classes Financial Market and Regulation are set in an arbitrary way, i.e. we defined the time instants of their state switch.

\section{if (Visibility is absent)}

then Products units move from the Institutions to the Investors with rate:

$\lambda_{\text {exchange }}=R{ }^{*} I C($ Crisis Level $) * G$

else Products units move from the Institutions to the Investors with rate:

$\lambda_{\text {exchange }}=R f * I C($ Crisis Level $){ }^{*} G * R$ the applicability of Mean-Field Analysis Model. The potentiality of this methodology can be resumed in three relevant features: flexible modeling approach, capacity of solving complex system, managing financial issues.
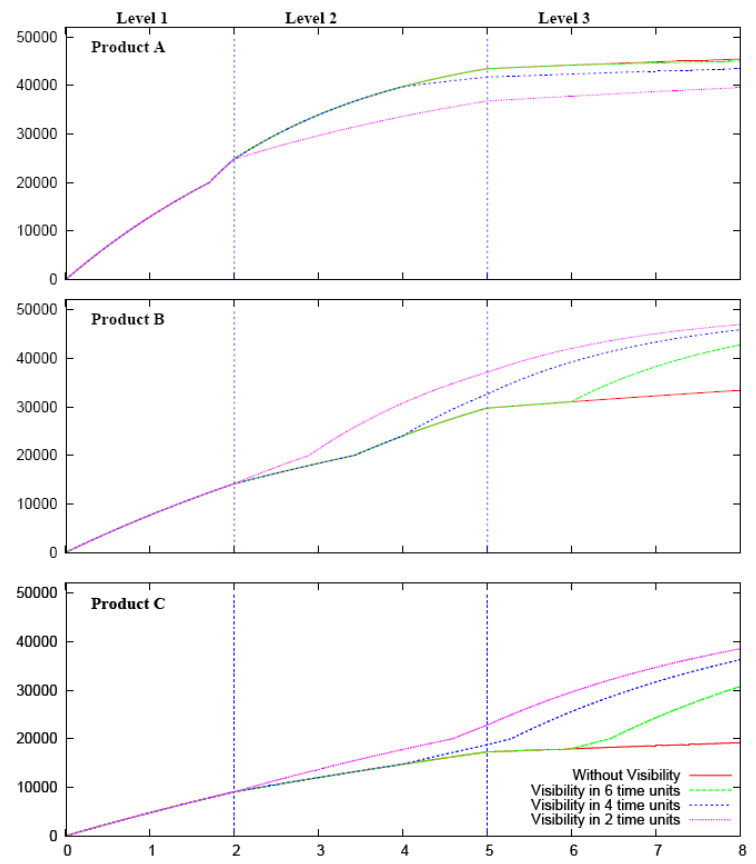

Fig. 3. The time evolution of the number of exchanged asset units for each product.

The flexibility of the approach can also be seen from the function and parameter levels. In fact, once the classes of the model have been selected, the use of different sets of functions and/or parameters allows to investigate various dynamics of interactions among actors. Indeed in this work we mainly focus on the regulation visibility impact on exchange rate of asset from bank to investor, but a further model refinement can be also focused on other important issues such as other type of regulations on products or authority.

The graphs reported in Fig. 3 show the diffusion of three products in different scenarios characterized by different level of crisis and by presence or absence of visibility. The $\mathrm{Tg}$ threshold was fixed to 20.000 , indeed, the plots point out that products increase their exchange rate when this value is exceeded.

In financial situation without visibility the product exchanges (see continuous red curves in Fig. 3) are strongly affected by the crisis level:

- Level 1: there is not crisis and the products A, B and C are exchanged into the market with different rates that depend on their risk factor (Rf);

- Level 2: the crisis is starting and exchange rates of the products $\mathrm{A}, \mathrm{B}$ and $\mathrm{C}$ tend to diminish according to the investment capacity reduction;

- Level 3: the crisis level is increased and exchange rates of the products A, B and C are reduced drastically due to the investment capacity reduction.

The third scenario (Level 3) draws attention on the liquidity crisis that invests not only the toxic product but also the products with a low effective risk that is perceived as high due to the impact of financial crisis. As outlined in the graphs all the three products reduce almost to zero their

In this section we present a qualitative result to explain 
exchange when entering into the level 3.
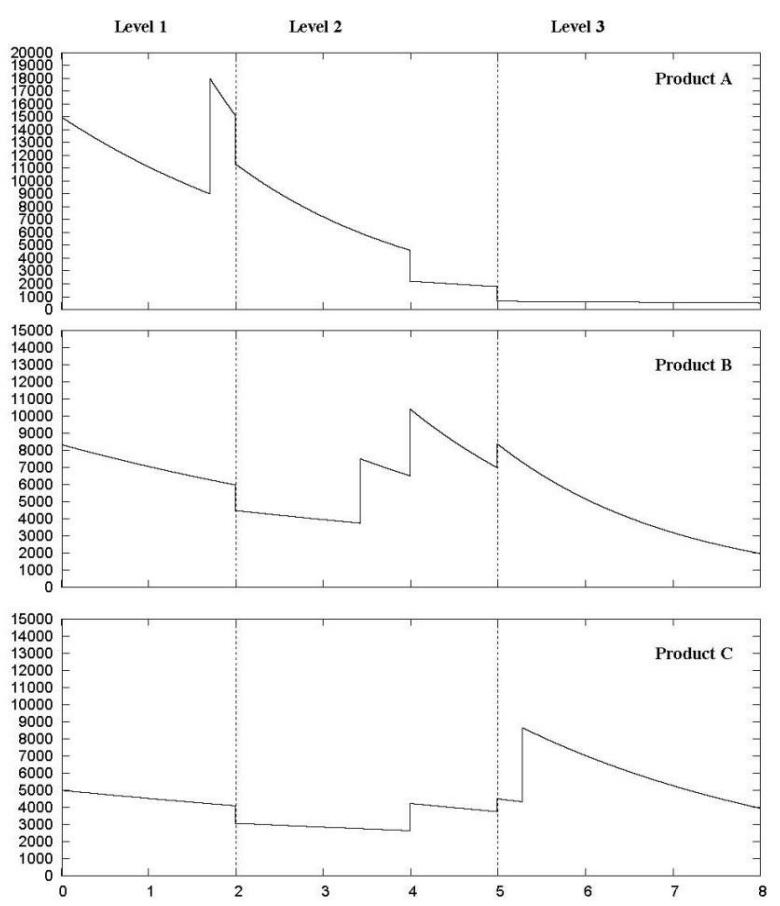

Fig. 4. The time derivative of the amount of sells of three products. The regulation is applied after 4 time units.

The application of the regulator policy modifies the qualitative results in the levels 2 and 3. The plots reported in Fig. 3 point out the effect of the regulation (dashed curves) applied at different time instants: after 2, 4, and 6 time units. When the market enters the crisis (level 2) -as well as the investment capacity decreases for all the system- the actors continue to exchange the products $\mathrm{A}, \mathrm{B}$ and $\mathrm{C}$ with different rate depending on their risk. The exchange rate remains higher than without visibility also in the scenario of high crisis (level 3) in which the exchange of the products B and $\mathrm{C}$ continues, whereas the exchange of the product $\mathrm{A}$ is strongly limited (but not stopped).

TABLE II: REGULATOR AND INVESTMENT CAPACITY FACTORS

\begin{tabular}{|c|c|c|c|}
\hline Level & R for toxic assets & $\begin{array}{c}\text { IC for toxic } \\
\text { assets }\end{array}$ & $\begin{array}{c}\text { IC for no } \\
\text { toxic assets }\end{array}$ \\
\hline 1 & 1 & 1 & 1 \\
\hline 2 & 0.3 & $1 / 3$ & $2 / 3$ \\
\hline 3 & 0.1 & $1 / 5$ & $4 / 5$ \\
\hline
\end{tabular}

As we showed in Fig. 4 is possible to follow the products diffusion rate by computing time derivative of the amount of sells. This plot reports the change rate of the three products when the regulation is applied after 4 time units. It can be noticed again that in Level 3 of crisis the exchange rate of Product $\mathrm{A}$ is reduced whereas the other product exchanges continue even with a lower rate due to the investment capacity reduction.

Thanks to the Mean Field Analysis model is possible to explain that the visibility on financial products avoid the liquidity crisis due to the informative asymmetry typical of the financial market. In fact, the end of the crisis has demonstrate that the regulators doesn't have the fundamental information for the supervision activities just think about the Otc derivative market for understanding the impossibility of keep the interconnected risk inside the financial actors. If the market is able to limit liquidity crisis then it could also limit the crisis consequences on macro level (think about the U.S.A. liquidity injection). The regulation control could be done by the authority creating a risk licence that takes the asset during all its market life.

In this work the financial crisis is considered as a discontinuous event. Indeed, the study of financial regulation can be applied in scenarios characterized by different events so dramatic that many of the rules driving the strategic behaviour of firms and governing the industry cease to continue. Events in the environment that could cause discontinuous environmental change include major technological breakthroughs, major changes in the laws and regulations that govern an industry (deregulation of the airline industry would be one example of this), a sudden change in the economy such as the stock market crash before the Great Depression, new sources of competition, or any combination of these events.

\section{ACKNOWLEGMENT}

Thanks to Marco Toledo and Mauro Filacchione of Tosetti Value Sim, a leading European Family Office, for their support.

\section{REFERENCES}

[1] R. D. Congleton, "On the Political Economy of the Financial Crisis and Bailout of 2008-2009," Public Choice, vol. 140, pp. 287-317, no. 3/4, September, 2009.

[2] X. Gabaix, A. Krishnamurthy, and O. Vigneron "Limits of Arbitrage: Theory and Evidence from the Mortgage-Backed Securities Market," Journal of Finance, vol. 62, no. 2, pp. 557-595, April., 2007.

[3] J. Duarte , "The Causal Effect of Mortgage Refinancing on Interest Rate Volatility: Empirical Evidence and Theoretical Implications," The Review of Financial Studies, vol. 21, no. 4, pp. 1689-1731, July, 2008.

[4] K. Gerardi, A. Lehnert, S. M. Sherlund, P. Wille, "Making Sense of the Subprime Crisis," Brookings Papers on Economic Activity, vol. 2008, pp. 69-145, 2008

[5] M. Bobbio, M. Gribaudo, and M. Telek, Analysis of large scale interacting systems by mean field method, in 5th International Conference on Quantitative Evaluation of Systems - QEST2008, St. Malo, pp. 215-224, 2008.

[6] T. G. Kurtz, Strong Aproximation Theorems for Density Dependent Markov Chains, Stochastic Processes and their Applications, vol. 6, 223-240, 1978.

[7] L. Papademos, "Strengthening macro-prudential supervision in Europe," Speech at the conference on After The Storm: The Future Face of Europe's Financial System organized by Bruegel, National Bank of Belgium and the International Monetary Fund Brussels, 24 March 2009.

[8] A. G. Haldane, "Rethinking the Financial Network", Speech delivered at the Financial Student Association, Amsterdam, April 2009

[9] L. H. Meyer, "The Challenges of Global Financial Institution Supervision," Remarks at the Federal Financial Institutions Examination Council, International Banking Conference, Arlington, VirginiaMay 31, 2000.

[10] I. W. Marsh and I. Stevens, Large Complex Financial Institutions: Common Influences on Asset Price Behaviour?, in Bank of England Quarterly Bulletin December 2003. 